\title{
Análisis discursivo del protocolo en la prensa española
}

\section{Analysis of protocol's discourse through Spanish press}

\author{
Elena Borau Boira. Universidad Camilo José Cela
}

Recibido: 5-II-2014 - Aceptado: 16-VI-2014

Resumen:

El protocolo, desde sus inicios, se ha ido adaptando a la gran transformación que ha ido experimentando nuestra sociedad y se ha modernizado hacia un mayor funcionalismo y practicidad para ir aportando las soluciones que la realidad va requiriendo. Un protocolo cada vez más simple y efectivo que ha ido dejando atrás la pompa y el boato de antaño. Aun así los medios de comunicación no reflejan esta realidad y siguen representando el protocolo como algo "anclado en el pasado", casi como obsoleto. Por lo tanto, el objetivo de este artículo es poner de manifiesto la negativa realidad que vive el protocolo en la prensa de nuestro país, para manifestar, en último término, la perjudicial influencia de esta representación para la imagen que esta disciplina tiene en nuestra sociedad. La representación que del protocolo hace la prensa es esencial para una correcta percepción del mismo. Tras llevar a cabo un riguroso análisis del discurso, se pondrán de manifiesto las negativas connotaciones del discurso del protocolo en la prensa, con sus desfavorables consecuencias para la percepción del mismo en la sociedad. En este artículo, por tanto, se reivindica la importancia del binomio protocolo-medios de comunicación, imprescindible para una buena gestión de la comunicación.

Palabras Clave:

Protocolo, medios de comunicación, prensa, análisis del discurso, representación mediática.

Abstract:

The protocol, from its beginning, has been adapting to big transformation that it's been experience our society and it became to more usefulness. Even though, The Mass Media don't reflect this reality and keep reflecting the protocol as something clings in the past, almost like something "old". In this article, I try to show up the negative reality that it lives in The Spanish Press, to express the harmful influence that has for the protocol's image in our society. This tool performs a great work in so many fields, but we can't forget the Mass Media's influence. Therefore, this rigorous discursive analysis will show up the negatives connotations of discourse of protocol in Spanish press, with its unfavorable final results. That is way, in this work I defend the importance the pairing of protocol and Mass Media, indispensable to a correct management of communication.

Key words:

Protocol, mass media, press, discurse analysis, media representation. 


\section{Introducción}

En la actualidad, el protocolo ha cambiado el objetivo y el destinatario de la comunicación que, gracias a la acción y colaboración de los medios informativos, se ha convertido en una comunicación de masas, que ofrece al ciudadano la actividad oficial de aquellas autoridades elegidas democráticamente y que ocupan un rango o puesto determinado en función de la representación que ostentan. Si, por otro lado, tenemos en cuenta que las normas y la organización protocolaria contribuyen a facilitar y encauzar la comunicación en la sociedad, no es de extrañar que también las empresas, grandes y pequeñas, como la sociedad civil en su conjunto, reclamen, cada vez con mayor interés, el uso del protocolo en sus actividades a través de los medios de comunicación de masas, a la audiencia con la que se les interese comunicar.

Hoy por hoy, el protocolo conserva su carácter organizador de una determinada modalidad de comunicación social, pero el objetivo y el destinatario de esa comunicación han pasado a ser los mismos que los de los medios informativos, debido a la presencia de éstos durante la celebración de los actos públicos.

El protocolo en esta función rectora define la proyección externa de los actos y condiciona su imagen pública a través de los medios de comunicación social mediante un proceso comunicativo que permite la interacción entre la organización y su público.

La investigación se llevará a cabo a través de los medios de comunicación, más concretamente de la prensa española, por ser el reflejo más fiel de la sociedad actual. Asimismo, los resultados obtenidos de la prensa española serán extrapolables a la sociedad actual ya que, partimos de la premisa de que "el proceso de la construcción social de la realidad está completamente supeditado a la práctica productiva del periodismo.”, (Browne, Del Valle, Silva, Carvajal e Inzulza, 2001:17).

Se coincide, por tanto, con Marín Calahorro, $(1997: 20)^{1}$ cuando otorga la misma relevancia a la práctica del protocolo como a la difusión del mismo.

La aportación que hacía Otero Alvarado (2004) ${ }^{2}$ en el V Congreso Internacional de Protocolo en este sentido también es muy representativa, ya que reclamaba abordar la situación académica del protocolo desde una visión hasta entonces inexistente.

"Los departamentos de protocolo deben moverse dentro de dos lógicas: la lógica de la eficacia en el proyecto y la lógica de que sean valoradas y comprendidas por los públicos. La primera se construye desde el conocimiento y la práctica del protocolo; la segunda, mediante la difusión de sus contenidos y explicación de sus códigos".

2 Frivolización del término "protocolo", que continúa asociándose con la banalidad de la mera apariencia. Desconocimiento general subyacente del significado exacto y la importancia del protocolo. Identificación del perfil de los profesionales del ceremonial y el protocolo con apellidos ilustres, afinidad política o personal. 
Otro autor de referencia que realiza aportaciones en esta línea es Vilarrubias (2010:33) ${ }^{3}$ por ir muy acorde con nuestro objeto de estudio, lo que se podría llamar la "frivolización del protocolo".

Por otro lado, la prensa juega un papel fundamental en la configuración de la cultura de la sociedad, es un factor imprescindible en la sociedad contemporánea española y por lo tanto, también es determinante en cuanto a su percepción sobre el protocolo. Según Berrocal y Rodríguez (1998:15) ${ }^{4}$ los medios informan, forman y recrean.

En este sentido, Borau (2013:633) señala: "el tratamiento informativo que los medios hacen de un tema en concreto es determinante a la hora de influir en la percepción que la sociedad puede tener a cerca del mismo". Y es aquí donde nos adentramos en el verdadero conflicto que existe entre la prensa y el protocolo, y que tantas veces han denunciado los profesionales y estudiosos del protocolo.

En definitiva, lo que este manuscrito se propone es comprobar, con rigor metodológico, que la imagen que los medios representan de esta disciplina no se corresponde con la realidad actual del protocolo, como la gran herramienta de comunicación que aporta valor y eficacia en tantos ámbitos de nuestra vida cotidiana.

\section{Relación actual del protocolo con la prensa de nuestro país}

A pesar de la adaptación del protocolo a la sociedad actual, y de su mayor simplicidad para una mayor efectividad, la relación entre medios de comunicación y protocolo sigue siendo conflictiva, incluso en alguna ocasión, los profesionales del protocolo han manifestado sentirse "maltratados" por ella. Un claro ejemplo es la carta que Fuente (2012) ${ }^{5}$ publicó en $A B C$ en protesta de una noticia que este periódico había publicado una semana antes:

En este sentido, y apuntando a la relación que los medios de comunicación mantienen con el protocolo, cabe destacar la opinión de Batlle, San Juan, Sunyé (2007:99), “los profesionales de los medios de comunicación deberían recibir unos cursos de formación en protocolo". Laforet (1997:52) apuesta por lo mismo, afirmando que existe un desconocimiento entre dos sectores que deben trabajar con tanta coordinación, los gabinetes de protocolo y de prensa. Frente a lo cual, puntua-

3 "En nuestros días son corrientes las expresiones "Sin protocolo", “Saltarse el protocolo", “con Protocolo estricto”, o también en otro epígono "El Protocolo sólo interesa a las Monarquías” o bien, "es un tema superado por las Relaciones Públicas (...)”.

4 "Informan ya que reflejan aquella parte de la realidad que facilita un mejor conocimiento de las grandes cuestiones del presente. Forman al expresar su punto de vista sobre los acontecimientos que inciden en la vida del hombre, ayudándoles en una toma de conciencia, y recrean, es decir, entretienen".

5 "Decir que hay cosas que no se aprenden en el instituto, ni en casa, ni en la universidad, cuando se habla de la organización de comidas de trabajo, es desconocer absolutamente la realidad de la enseñanza oficial que se imparte en este grado (...). Quiero transmitir nuestra indignación también al referirse la autora a que las clases de protocolo van dirigidas únicamente a grandes empresarios, políticos y gente de la nobleza". 
liza el autor, "no sólo se palpa una inexistencia casi absoluta de cursos enfocados a los miembros de los medios de comunicación, sino que no existen manuales para su tarea como informadores".

Debemos ser conscientes, por tanto, de que estamos ante dos disciplinas destinadas a encauzar comunicación y que se caracterizan, sobre todo, por ser actividades técnicas y sociales. En esta misma línea, Laforet (1997a:39) apunta: "si miramos atrás, e incluso hoy en día, el concepto comunicación diverge mucho si se aplica a protocolo o periodismo, pues eran disciplinas que apenas convergían".

Tal y como señala Sierra $(2008: 345)^{6}$ investigaciones previas en este campo son escasas o más bien nulas. La prensa, especialmente la que se concibió con fórmulas próximas a las que perviven en la actualidad, aparece muy tardíamente en la historia de la humanidad, si lo comparamos con la actividad protocolaria, no ya en lo que se refiere a la organización y desarrollo de los más diversos ceremoniales, sino a la regulación, sistematización y oficialidad de las normas que regían y rigen la vida oficial de la esfera pública y privada.

\section{Hipótesis}

La formulación de unas hipótesis de partida es imprescindible para cualquier investigación que pretenda ser calificada de científica. Por lo tanto, y por tratarse de un trabajo con limitaciones de espacio, la hipótesis principal que se intentará corroborar será la siguiente:

La representación que la prensa española hace de los códigos del protocolo no se corresponde con su verdadera razón de ser.

Con esta hipótesis se intentará comprobar que los rasgos que la prensa atribuye al protocolo son, sobre todo, de rigidez, inflexibilidad y obligatoriedad, así como valorar hasta qué punto las connotaciones que se sustraen de su representación son, en general, negativas. De la misma forma, con esta hipótesis se pretende refutar que el protocolo que representa la prensa española está mayoritariamente relacionada sólo con actores de primer nivel (realeza, primeros mandatarios o personajes de muy reconocido prestigio).

\section{Objetivo}

Una vez corroborada esta hipótesis, estaremos en disposición de alcanzar el principal objetivo que se propone en este artículo: demostrar, con rigor metodológico, la negativa imagen que la prensa española representa sobre el protocolo.

6 "Son muchas las dudas y las incógnitas que quedan aún por resolver en una materia que tiene un extenso recorrido histórico, pero que ha estado olvidada desde planteamientos científicos". 
Se observará la relación terminológica que se da en la prensa sobre el protocolo, así como los rasgos y connotaciones que se sustraen de la imagen de esta disciplina en la prensa, o si necesita de un determinado nivel de actores para su representación en este medio.

\section{Metodología}

Para conseguirlo se llevará a cabo un análisis del propio discurso del protocolo en la prensa, con el fin de concretar cómo es su "realidad mediática" en este medio, y observar si corresponde con su verdadera razón de ser. Se tratará de un análisis en profundidad sobre la representación del protocolo en la prensa, donde se determinarán los parámetros que se dan en las noticias, desde el punto de vista de la interactividad de las partes. Se procederá a estudiar el protocolo en la prensa desde su perspectiva más profunda, es decir, el discurso desde el principio de autoridad, de actualidad, o proximidad. Factores en donde también influye la percepción que los periodistas y su editorial tienen sobre un tema para reflejarlo en sus páginas. Pero, ¿qué es un análisis del discurso?

La organización de la producción de las noticias tiene muchas dimensiones y niveles de descripción. Tuchman (1978) en Van Dijk (1990:22) realiza uno de los estudios más interesantes e innovadores acerca de la producción de las noticias, en donde "la noticia no se caracteriza como una imagen de la realidad sino como un marco a través del cual se construye rutinariamente el mundo social”. Para este autor, los reporteros se mueven dentro de una red que constituye un mecanismo organizativo estratégico que debe plegarse sobre las fuentes informativas tan efectivamente como sea posible. Las clasificaciones, según Tuchman, de los acontecimientos periodísticos, permiten a los reporteros asignar ciertos valores a tales sucesos, mientras que al mismo tiempo, les otorgan la libertad de negociar sobre su variación. Un microanálisis profundo exige, por tanto, descripciones sistemáticas de la noticia como producto de las prácticas de fabricación informativa.

Link (1983) en Wodak y Meyer (2003:63) define el discurso como "un concepto de habla que se encontrará institucionalmente consolidado en la medida en que determine y consolide la acción y, de este modo, sirva ya para ejercer el poder". En esta misma línea, para Wodak y Meyer (2003:63-66) ${ }^{7}$ los discursos no poseen interés por el hecho de ser expresiones de la práctica social, sino por el de contribuir a determinados fines, a saber, el de ejercer el poder a todos los efectos:

La semiótica actual también se ocupa del significado de los textos tal como lo hace el análisis de contenido, pero se diferencia de éste en que su unidad última de análisis es el signo. A través de estos signos la semiótica pretende fundamentalmente la construcción del texto más que su análisis y adopta todo un aparato descriptivo-interpretativo de las acciones de los sujetos.

\footnotetext{
"En los discursos, la realidad no queda simplemente reflejada, sino que poseen una "vida propia" que determina su relación con la realidad. (...).Y tampoco son realidades materiales de segunda clase ni elementos "menos materiales" que la realidad "real”. Los discursos son más bien realidades materiales con plena validez".
} 
En el presente artículo, el análisis descriptivo se centra en la investigación analítica del discurso dirigido a la comunicación de masas, a la explicación del discurso periodístico que conlleva una descripción de las estructuras textuales de la noticia, como una descripción de los procesos de producción y recepción del discurso periodístico en situaciones comunicativas y contextos socioculturales.

Siguiendo a Van Dijk (1990:249), también se considera la "noticia periodística como un tipo específico de discurso". La noción de noticia es ambigua, y por tanto, en los medios implica los siguientes conceptos:

- $\quad$ Nueva información sobre sucesos, objetos o personas.

- $\quad$ Un programa tipo (de televisión o radio) en el cual se presentan ítems periodísticos.

- Un ítem o informe periodístico, como por ejemplo un texto o discurso en la radio, en la televisión o en el diario, en el cual se ofrece una nueva información sobre sucesos recientes.

Este trabajo se centra en las noticias como discurso tal como es expresado, utilizado o hecho público en los medios periodísticos o los medios de información pública tales como la prensa. Se trata, en resumen, de la nueva información tal como la proporcionan los medios y tal como la expresan los informes periodísticos.

Asimismo, se estudia la noticia en la prensa, es decir, el discurso o los artículos periodísticos publicados cotidianamente en los diarios, ya que desempeñan un rol crucial en la comunicación de masas, y no sólo en nuestras propias sociedades occidentales, sino también en las sociedades donde la televisión es aún un bien escaso y existen pocos estudios acerca del discurso de la noticia periodística.

Este análisis considera el discurso de los medios, y por lo tanto también el de la noticia como una forma particular de práctica social e institucional. Esta práctica discursiva de la producción o de la recepción de la noticia puede analizarse en dos componentes principales: un componente textual y un componente contextual. En el presente artículo se analizará el primero: las diferentes estructuras del discurso periodístico a través de diferentes categorías.

El discurso, por tanto, que interesa en este trabajo es aquel que es potenciado en términos de construcción social de la realidad por los propios medios de comunicación. Concibiendo, por supuesto, que los medios de comunicación son las mejores herramientas que tiene la sociedad contemporánea para reproducir la ideología del consenso y del poder, ya que construyen estructuras interpretativas que anuncian qué y cómo se debe comprender la realidad y el día a día que le da vida y sustento.

La complejidad que conlleva esta conjugación discurso-sociedad permite dilucidar cómo las construcciones mediáticas afectan directamente a la conformación identitaria de comunidades en general, que en este trabajo se focalizan en el propio discurso del protocolo en la prensa española. De este análisis obtendremos unos resultados que nos ayuda- 
rán a determinar la imagen que del protocolo proyecta la prensa española para vislumbrar la influencia que ejerce en la sociedad.

\subsection{Diseño de la Investigación}

Con el fin de estudiar el protocolo en la prensa desde su perspectiva más profunda, es decir, el discurso desde el principio de autoridad, de actualidad, proximidad, novedad, o impacto emocional del tema, se establece una clasificación clara y concisa basada, principalmente, en la muestra y las variables de selección escogidas.

\subsection{Muestra para el análisis}

Para alcanzar el objetivo antes propuesto, de una forma rigurosa y contrastable, se eligen todas aquellas noticias publicadas en los tres periódicos de mayor tirada y de información general, de nuestro país: El País, El Mundo y ABC, en las que se hable o contengan la acepción "protocolo-ceremonial” durante el año 2010, es decir 309 noticias.

Se han seleccionado los tres periódicos más importantes de pago y de tirada nacional del país según la Oficina de Justificación de la Difusión y el Estudio General de Medios (OJD y EGM) ${ }^{8}$ : El País, El Mundo y ABC, que representan la referencia informativa de nuestro país y, por lo tanto, son fiables indicadores y extrapolables a la prensa escrita en general. Asimismo, nos centraremos en la acepción protocolo-ceremonial frente a otras contempladas en la vigésima segunda edición de la Real Academia Española9: "Regla ceremonial diplomática o palatina establecida por decreto o por costumbre”.

\subsection{Variables de selección para el análisis discursivo}

Se analizarán las variables considerándolas de manera individual, por ello es especialmente relevante una sistematización adecuada de las mismas. Se trata de un análisis descriptivo y profundo de cada variable a estudiar. Las variables que componen el presente análisis del discurso son las siguientes:

8 Página web del Estudio General de Medios. Disponible en http://www.aimc.es/-Datos-EGM-Resumen-General-.html. Abril 2010 marzo 2011. Consultado 28-12-13.

9 Página web RAE. Disponible en http://buscon.rae.es/draeI/SrvltGUIBusUsual?LEMA=protocolo. Consultado el 12-02-12. 


\subsubsection{Clase de protocolo}

Puesto que en todas las noticias a analizar aparecerá el término protocolo, en el presente ítem se analizará la clase de protocolo a la que corresponde. Se fundamentará en la definición de Marín Calahorro (1997:30 $)^{10}$ de protocolo, por su sencillez y claridad.

\subsubsection{Contexto}

Se dividirá el protocolo en dos tipos fundamentales:

Nacional: Cuando el contexto del discurso se desarrolle dentro del país.

Internacional: Cuando sus actores sean extranjeros o tenga lugar fuera del territorio patrio.

\subsubsection{Nivel del hecho}

El nivel del hecho a analizar vendrá determinado por el nivel de los actores que intervienen:

Nivel 1: Hecho en el que interviene personas pertenecientes a la Realeza o el Papa como máximo representante de la Iglesia.

Nivel 2: Intervienen políticos o personas de clase social alta.

Nivel 3: Actúan ciudadanos de clase social media.

Nivel 4: Hecho que versa sobre personas de clase social baja.

\subsubsection{Relación terminológica}

Con esta variable identificaremos los rasgos o peculiaridades que, en cada caso, la prensa atribuye al protocolo en el discurso. Para ello, se ha establecido una clasificación de siete categorías para determinar las relaciones terminológicas más utilizadas en la prensa española a la hora de representar el protocolo. La clasificación se ha creado en base al propio trabajo de campo del análisis, en el que se han identificado las más utilizadas, de manera que todas y cada una de las noticias pertenezcan a una categoría:

10 1. Esfera pública: en ella las actividades sociales se estructuran y formalizan con dimensión pública. Pueden ser: 1.1.1. Oficial: Perteneciente a las altas instituciones del Estado y por las diferentes ramas de la Administración.

1.1.2. No oficial: Entidades cuyas actividades tienen relevancia e interés para amplios sectores de la sociedad.

2. Esfera privada: actividades de carácter social que no trascienden a un público masivo.

1.1.3. Formal: Se mantienen ciertas formalidades: Una boda, bautizo, comunión, exequias, etc.

1.1.4. Informal: Priman las muestras de afecto sobre el respeto a determinadas formas: Reuniones de amigos, familia. 
1. Correcta representación del protocolo

2. Cumplir el protocolo

3. Protocolo como puro formalismo

4. Protocolo relacionado con gastos

5. Rigidez protocolaria

6. Romper el protocolo

7. Se atribuye al protocolo algo que no le corresponde

Esta categoría nos llevará a determinar cuáles son las relaciones terminológicas más frecuentes con las que la prensa relaciona el protocolo, las características y los rasgos que le atribuye, por lo que se trata de la categoría más determinante del estudio.

\subsubsection{Connotación}

Este ítem está dirigido a estudiar la connotación que se sustrae del protocolo de su relación terminológica y representación en la noticia. Se ha creado en base a nuestro criterio, como investigadora del protocolo y se ha establecido tres clases de connotación:

- $\quad$ Negativa: La noticia le adjudica al protocolo unos valores que se perciben como negativos.

- $\quad$ Positiva: La noticia le otorga unos rasgos favorables al protocolo.

- $\quad$ Neutral: La noticia menciona el término pero no existe atribución alguna para el protocolo.

\section{Resultados}

Tras realizar el análisis del discurso de las noticias que componen la muestra, es decir, las 309 noticias publicadas durante el año 2010 que hablan o contienen la acepción de protocolo-ceremonial que estamos estudiando, se procederá a explicar los resultados.

Con el análisis del discurso se ha fragmentado el corpus en varios elementos significativos para la investigación, de modo que nos permitan establecer categorías significativas a la hora de determinar la relación terminológica que la prensa española hace del protocolo. 


\subsection{Clases de Protocolo}

Tabla I. Tabla de frecuencia: Contexto geográfico

\begin{tabular}{|l|l|c|c|c|c|}
\hline & & Frecuencia & Porcentaje & Porcentaje válido & Porcentaje acumulado \\
\hline \multirow{5}{*}{ Válidos } & Privado, formal & 55 & 17,8 & 17,8 & 17,8 \\
\cline { 2 - 6 } & Privado, informal & 2 &, 6 &, 6 & 18,4 \\
\cline { 2 - 6 } & Público, no oficial & 44 & 14,2 & 14,2 & 32,7 \\
\cline { 2 - 6 } & Público, oficial & 208 & 67,3 & 67,3 & 100,0 \\
\cline { 2 - 6 } & Total & 309 & 100,0 & 100,0 & \\
\hline
\end{tabular}

Fuente: Elaboración propia

Se observa que el tipo de protocolo que más a menudo refleja la prensa española (frecuencia) es "Público, oficial", es decir, el perteneciente a las altas instituciones del Estado y las diferentes ramas de la Administración, con un 67,3\% del total, seguido con una gran diferencia el "Privado, formal” con un 17,8\%. Asimismo, vemos cómo la categoría "Privado, informal”, pasa casi desapercibida en la prensa española, con un 0,6\% y la tercera categoría en presencia en la prensa española es "Público oficial" con un $14,2 \%$.

\subsection{Contexto}

Tabla II. Tabla de frecuencia: Contexto geográfico

\begin{tabular}{|l|l|c|c|c|c|}
\hline & & Frecuencia & Porcentaje & Porcentaje válido & Porcentaje acumulado \\
\hline \multirow{4}{*}{ Válidos } & Internacional & 101 & 32,7 & 32,7 & 32,7 \\
\cline { 2 - 6 } & Nacional & 208 & 67,3 & 67,3 & 100,0 \\
\cline { 2 - 6 } & Total & 309 & 100,0 & 100,0 & \\
\hline
\end{tabular}

Fuente: Elaboración propia

Se comprueba que del total de la muestra analizada, es mayoría el contexto nacional para hablar de protocolo con un $67,3 \%$ frente al $32,7 \%$ del contexto internacional. 


\subsection{Nivel del hecho}

Tabla III. Tabla de frecuencia: Nivel del hecho

\begin{tabular}{|l|l|c|c|c|c|}
\hline & & Frecuencia & Porcentaje & Porcentaje válido & Porcentaje acumulado \\
\hline \multirow{5}{*}{ Válidos } & Nivel 1 & 108 & 35,0 & 35,0 & 35,0 \\
\cline { 2 - 6 } & Nivel 2 & 196 & 63,4 & 63,4 & 98,4 \\
\cline { 2 - 6 } & Nivel 3 & 5 & 1,6 & 1,6 & 100,0 \\
\cline { 2 - 6 } & Total & 309 & 100,0 & 100,0 & \\
\hline
\end{tabular}

Fuente: Elaboración propia

Vemos que el nivel 2, es decir, el compuesto por políticos o personas de clase social alta, es el más representado en la prensa española a la hora de hablar de protocolo con un 63,4\%: políticos, empresarios de prestigio, deportistas de élite, etc.

A continuación encontramos el nivel 1 con un 35\%: personas de la realeza española y también europea, y el Papa como el máximo representante de la Iglesia. Asimismo, vemos cómo el nivel 3 pasa casi desapercibido con un 1,6\% (sociedad clase media) y el nivel 4 no tiene ningún tipo de presencia (ciudadanos clase baja).

\subsection{Relación terminológica}

Esta categoría es la más determinante del presente estudio, ya que establece los términos, características y atributos con los que se relaciona al protocolo en el discurso mediático. Para conseguirlo, se creado una clasificación, basada en la experiencia del trabajo de campo que se ha llevado a cabo para analizar el discurso de las noticias que versan sobre protocolo, de manera que todas pertenecen a alguna de los siete tipos. Por tratarse de la categoría más representativa del análisis del discurso, se llevará a cabo igualmente una comparativa entre los tres periódicos para observar la relación terminológica que el periódico hace cada del protocolo.

Se ha creado esta clasificación "a medida” con la intención de agrupar la relación terminológica en las categorías que más se repiten en la prensa española a la hora de tratar el protocolo, para poder observar cómo representa la prensa dicha disciplina, con qué la relaciona terminológicamente y qué características o rasgos se le atribuyen. 
Tabla IV. Tabla de frecuencia: Relación terminológica

\begin{tabular}{|l|l|c|c|c|c|}
\hline & & Frecuencia & Porcentaje & Porcentaje válido & Porcentaje acumulado \\
\hline \multirow{5}{*}{ Válidos } & Correcta representación del protocolo & 90 & 29,1 & $\mathbf{2 9 , 1}$ & 29,1 \\
\cline { 2 - 6 } & Cumplir el protocolo & 13 & 4,2 & 4,2 & 33,3 \\
\cline { 2 - 6 } & Protocolo puro formalismo & 21 & 6,8 & 6,8 & 40,1 \\
\cline { 2 - 6 } & Protocolo relacionado con gastos & 34 & 11,0 & 11,0 & 51,1 \\
\cline { 2 - 6 } & Rigidez protocolaria & 47 & 15,2 & 15,2 & 66,3 \\
\cline { 2 - 6 } & Romper el protocolo & 38 & 12,3 & 12,3 & 78,6 \\
\cline { 2 - 6 } & $\begin{array}{l}\text { Se atribuye al protocolo algo que no le } \\
\text { corresponde }\end{array}$ & 66 & 21,4 & 21,4 & 100,0 \\
\cline { 2 - 6 } & Total & 309 & 100,0 & 100,0 & \\
\hline
\end{tabular}

Fuente: Elaboración propia

La categoría "Correcta representación del protocolo" representa todas las noticias en las que se hace un correcto uso del protocolo. Con un porcentaje del $\mathbf{2 9 , 1 \%}$ es la categoría que más presencia tiene de las siete, aunque está muy lejos de ser óptimo, ya que significa que alrededor del 70\% restante está compuesto por las noticias publicadas en la prensa española que no hacen una correcta representación del protocolo. Esta categoría reúne aquellas noticias que representan el protocolo como una herramienta que ayuda en la organización, que facilita el trabajo, que comunica y/o que otorga la relevancia necesaria a un acto determinado.

De cada categoría se van a extraer algunos de los ejemplos más significativos que se han encontrado a través del trabajo de campo, para una mejor comprensión. En la mayoría, se trata de un ejemplo de cada categoría por cada periódico, aunque al no encontrar ejemplos de cada uno para todas las variables, se han escogido aquellos más significativos y que mejor la ejemplifican.

El protocolo aporta valor:

"Los expertos en protocolo de la Casa Blanca se apresuraron a aportar valor a esa escena" (ABC, 23-02-10. Pp.08).

"Una vez más, se excluye al protocolo que hace posible el diálogo de la verdad" (El País, 29-07-10.Pp.13).

El protocolo aporta soluciones: 
- Con la prensa es más fácil porque hay un determinado protocolo” ( $A B C, 20-09-10$. Pp.89).

"El protocolo evitó que la visita del presidente sudafricano se convirtiera en un problema" (El Mundo, 21-03-10. p.16).

El protocolo que comunica:

"Esta ruptura del estricto protocolo de las graníticas tradiciones demuestra la gravedad del momento" (El País, 0504-10.Pp.28).

- El carácter insólito del protocolo que marcó su despedida, mostró la deliberada confusión sobre el papel político de Kirchner en Argentina” (El País, 29-10-10.Pp.30).

6.4.1. La categoría que le sigue con un $21,4 \%$ es "Se atribuye al protocolo algo que no le corresponde", es decir, se le adjudican características o responsabilidades que no pertenecen al ejercicio propio del protocolo

Se observa cómo la prensa española recurre, muy a menudo, al sarcasmo, atribuyendo fallos que no corresponden o incluso estridencias de los protagonistas de la noticia, al protocolo cuando los mismos periodistas son conscientes de que no se trata precisamente de protocolo. Ironizan con temas de los que resulta incómodo hablar abiertamente, frivolizando el protocolo y haciéndole un flaco favor, ya que desvirtúan negativamente su verdadera razón de ser. Asimismo, la frivolización a la que, a menudo, es sometido nuestro objeto de estudio, se incluiría también en esta categoría, es decir, cuando se asemeja el protocolo con glamour, lujo y ostentación.

Otra forma de atribuir rasgos que no se corresponden con el ejercicio de esta disciplina es la de emplear términos confusos para referirse a ella. Vemos algunos de los ejemplos más representativos:

Se atribuye al protocolo algo que no le corresponde:

- $\quad$ Alguien ¿ de protocolo? decidió por su cuenta que el infante no estaba invitado ni estaría bien visto por el Rey". ( $E l$ Mundo 28-03-10.Pp.16).

- Sorprendió en Estocolmo e incluso fue interpretado como un error de protocolo". (El País 24-06-10.Pp.38).

Términos confusos:

"Protocolo expansivo" (Como forma de marketing o propaganda). (ABC, 12-09-10. Pp.96).

“Casa de protocolo” (En lugar de casa de visita) (El Mundo, 17-02-10.Pp.20).

"Partidos de protocolo" (En lugar de partidos de fútbol amistosos) (El País, 19-11-10.Pp.59). 
Se han encontrado otros muchos casos que muestran esta confusión terminológica, por temas obvios de espacio se expondrán sólo aquellos más llamativos:

- "Se recortarán gastos para la organización de las fiestas patronales, los actos de protocolo y publicidad" (Los actos de protocolo no existen). (El País, 12-06-10.Pp.02).

"Acabar también con los eventuales, con los gastos de protocolo y dietas" (Los gastos de protocolo no existen). (ABC, 14-05.10.Pp.14).

6.4.2. "Rigidez protocolaria" es la tercera categoría con un $15,2 \%$, lo que significa que una gran cantidad de noticias hablan del protocolo como algo rígido, inflexible o distante

Muchas noticias hablan del protocolo como lo que impide a los protagonistas ser cercanos y espontáneos, algo que es totalmente falso y que contribuye a crear esa negativa percepción del protocolo en la prensa. Hemos visto cómo este medio refleja el protocolo como lo que impide ser más humano en un determinado momento, expresivo o incluso mostrar ningún tipo de muestra de cariño, algo que, evidentemente no es cierto. Asimismo, se observa la recurrente relación entre protocolo y poder, al que parece que va unido inexorablemente en la prensa española.

Rigidez protocolaria:

"Ya en Valencia, concluido el acto institucional y sin las rigideces del protocolo..." (ABC, 19-12-10.Pp.33).

"El protocolo del acto encontró un respiro cuando a Raúl se le cayó la medalla” (El Mundo, 25-05-10.Pp38).

Protocolo VS Cercanía

- $\quad$ "En la plaza de la Catedral el Papa fue recibido por miles de fieles, a los que saludó fuera de protocolo" (ABC, 11-0710. Pp.10).

- “Todo era protocolo hasta que Del Bosque sacó a Aragonés a compartir con todos la foto y el momento” (El Mundo, 23-10-10.Pp.56).

- No había lágrimas, el protocolo lo invadía todo" (El País, 23-04-10.Pp.62).

6.4.3. La cuarta categoría con mayor presencia es "Romper el protocolo" con un $12,3 \%$, lo que supone que la prensa española recurre demasiado a menudo a esta expresión para referirse al protocolo

Esta categoría está muy relacionada con la anterior "Rigidez protocolaria", ya que el trasfondo es el mismo, pero utiliza literalmente ésta expresión: 
- La reina rompió el protocolo en un gesto divertido y cercano al ciudadano” (ABC, 11-07-10. Pp.15).

“El hijo del técnico español rompió el protocolo y se convirtió en una de las estrellas de la fiesta” (El Mundo, 08-0710.Pp.92).

Vemos cómo la prensa utiliza este recurso de forma muy habitual, el de resaltar la amabilidad del protagonista de la noticia a costa de "romper el protocolo", cuando realmente no hace falta romper nada para mostrarse con naturalidad y sentido común.

6.4.4. La categoría "Protocolo relacionado con gastos" es la quinta en presencia con un $11 \%$, lo que supone una importante relación entre esta disciplina con gastos, bien con recortes, bien con derroche económico o bien con corrupción

Esta relación terminológica es evidentemente negativa pero lamentablemente frecuente, creando una percepción más que errónea en la sociedad. En muchas ocasiones los gastos de protocolo se asemejan en la prensa a los generados por las fiestas del pueblo o de la ciudad, a copiosas comidas, viajes o móviles, entre otros gastos más que prescindibles. Se trata de un símil nefasto para el protocolo porque provoca que se vea en nuestra sociedad como un gasto superfluo e innecesario, lo cual está mal, pero peor está la propia definición, ya que los "gastos de protocolo" por sí mismos, no existen.

De la misma forma, se observa cómo los imputados o sospechosos de corrupción han atribuido sus excesos económicos a "gastos de protocolo", o también vemos cómo la prensa muchas veces denuncia el derroche económico que llevan a cabo determinados políticos o grupos políticos, culpando al protocolo como causa de tal derroche. Muchas son las noticias que nos hemos encontrado de este estilo y mucho el daño que han causado y siguen causando a nuestro objeto de estudio, contribuyendo a que la sociedad lo perciba como un gasto innecesario y totalmente prescindible. Además, esta muestra la componen noticias publicadas en el año 2010, por lo que, a día de hoy esta relación ha aumentado por el empeoramiento de la situación económica y la continua publicación de noticias sobre la crisis, recortes, ajustes, despidos, corrupción, etc.

Protocolo como gasto innecesario:

- "Drástico recorte del 70\% en gastos de publicidad, actos lúdicos y recreativos, conferencias, protocolo y telefonía" (ABC, 07-03-10. Pp.30).

- $\quad$ "Antes que recortar en presupuesto público habría que hacerlo en gastos como el protocolo o propaganda" ( $E l$ Mundo, 20-05-10.Pp.03).

Protocolo relacionado con corrupción: 
- $\quad$ Concejal de IU: "Si es Arenas quien se come la mariscada, entonces es protocolo o algo normal" (ABC, 30-1010.Pp.22).

- $\quad$ “Gastos de protocolo como innumerables sobornos, gastos, viajes, etc" (El Mundo, 07-04-10.Pp.01).

6.4.5. La sexta categoría es la que relaciona el protocolo con "Puro formalismo", es decir, que lo representan más como un estorbo que como una ayuda, con un 6,8\% del total de la muestra

El protocolo, en esta categoría, viene relacionado con la excesiva burocracia o lentitud propia de las administraciones o instituciones. Todas las noticias que se engloban en esta categoría denotan lo mismo: excesiva inflexibilidad, formalismo o burocracia del protocolo, presentándolo como algo frío y distante en la prensa.

Protocolo visto como un estorbo:

- $\quad$ "Sigue comportándose con la misma naturalidad de antes de tener una jefa de protocolo" (El Mundo, 1310.10.Pp.06).

- Es un hombre comunicativo, el protocolo está reducido a su mínima expresión" (El País, 14-02-10.Pp.14).

Protocolo como puro formalismo:

- "Al acto le sobró protocolo y séquito oficial. Seguramente Letizia hubiera querido una visita más cercana" (El Mundo, 18-09-10.Pp.18).

“El Rey intentará cautivar a sus interlocutores rebajando el nivel de formalidad y de protocolo" (El País, 06-1210.Pp.17).

6.4.6. En cuanto a presencia en la muestra analizada, está la formada por aquellas noticias que hablan del protocolo recurriendo a la tan manida expresión "Cumplir el protocolo", denotando esta obligación de la que tanto se habla en la prensa española para hablar de protocolo

Esta categoría está igualmente relacionada con las categorías "Rigidez protocolaria" y "Romper el protocolo", ya que la connotación es la misma, pero utiliza de forma literal la expresión "Cumplir el protocolo" o "Seguir el protocolo" como otra forma más de hablar del protocolo desde la rigidez y la inflexibilidad.

- Zapatero llegó más tarde que el Rey y Chacón no cumplió el protocolo con la indumentaria” (El Mundo, 07-0110.Pp.09).

- Seguía el protocolo a la perfección, parecía haber nacido con la lección aprendida” (El País, 14-02-10.Pp.40). 
Por último y como la categoría que cierra esta investigación habla de las connotaciones que se sustraen de nuestro objeto de estudio en las noticias, nos parece interesante exponer algún ejemplo de los casos más representativos en cuanto a connotaciones negativas:

- A veces conviene prestar oído al espíritu y dejar al lado el protocolo". (Dice Cristina Kirchner para justificar una de sus rarezas) (El Mundo, 23-05.10. Pp.31).

- $\quad$ “Acudieron siete mil jefes de protocolo y veinte mil mindundis del gabinete de la señora ministra” (ABC, 24-03-10. Pp.13).

Con esta explicación se ha tratado de ejemplificar, con expresiones literales de algunas de las noticias que forman la muestra analizada, los resultados que hemos obtenido en cifras, para visualizarlo de una forma verídica y contrastable.

Asimismo, y de forma que se visualicen todos estos datos correctamente, se ilustrarán con el correspondiente gráfico (Gráfico 1) para observar cómo es la relación terminológica con el protocolo en cada periódico estudiado, y de manera aproximada.

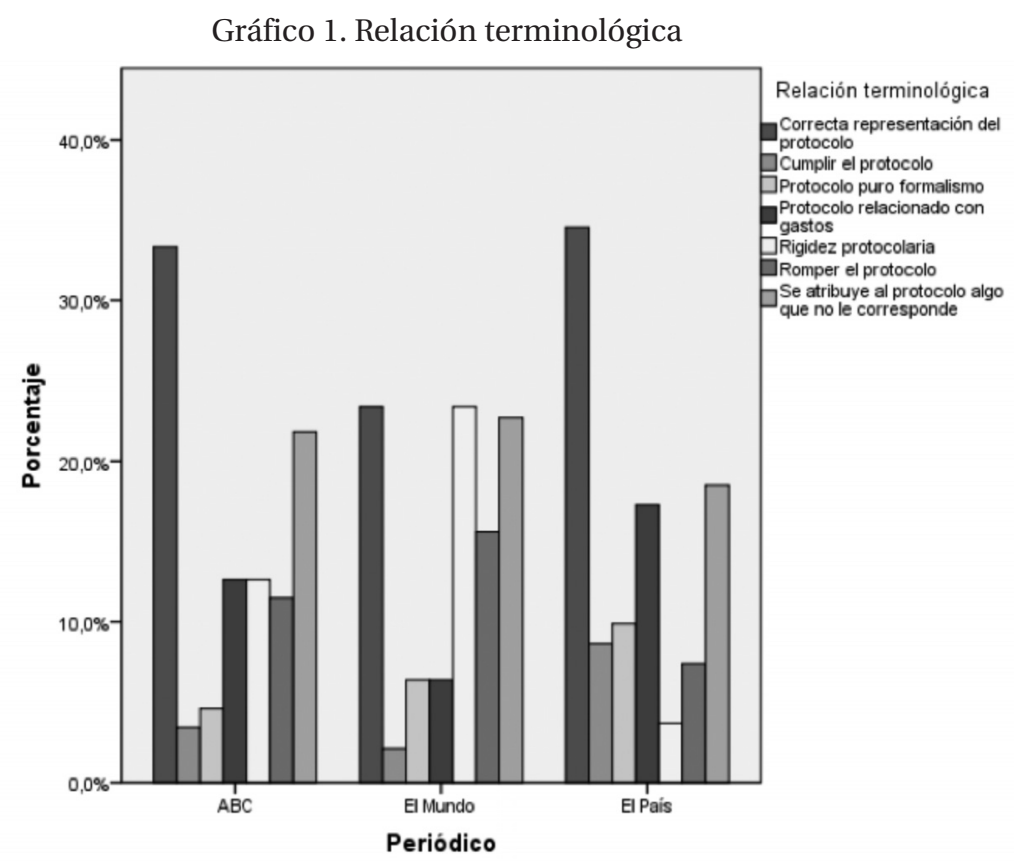

Fuente: Elaboración propia 
Explicación del gráfico:

- $\quad$ Correcta representación del protocolo:

El periódico El País es el que más presencia tiene en esta categoría con el 35\% aproximado, lo que significa que hace una correcta representación del protocolo por encima de $A B C$ (algo por encima del 30\%) y sobre todo de El Mundo, que se queda en último lugar con un $20 \%$ aproximado.

- Se atribuye al protocolo algo que no le corresponde:

El Mundo es el periódico que con más frecuencia atribuye al protocolo algo que no le corresponde, con un porcentaje mayor al $20 \%$, le sigue $A B C$ alrededor del $20 \%$ y, por último, es El País el diario que menos veces recurre a esta opción, quedando por debajo del $20 \%$.

- $\quad$ Rigidez protocolaria:

El Mundo es la cabecera que con más frecuencia recurre a este recurso para hablar de protocolo, con más de un $20 \%$ del total, le sigue $A B C$ con algo más de un $10 \%$ y en última posición encontramos a El País, que no llega al 5\%.

- $\quad$ Romper el protocolo:

Una vez más, El Mundo es el periódico que más veces recurre a esta expresión para hablar de protocolo, con más de un $10 \%$ del total, seguido de $A B C$ con algo por debajo del 10\% y por último El País, que no llega al 5\%.

- $\quad$ Protocolo relacionado con gastos:

El País es el periódico con mayor porcentaje en esta categoría, mayor al 10\%, seguido por $A B C$ y $E l$ Mundo, con una cuota en torno del $5 \%$.

- $\quad$ Protocolo como puro formalismo:

Ninguno de los porcentajes llegan al 10\% siendo El País el periódico con mayor presencia, seguido de El Mundo con alrededor de un 5\% y $A B C$ con algo menos.

- $\quad$ Cumplir el protocolo:

Aunque los porcentajes son los más bajos y por lo tanto, son menos representativos, El País es el periódico que más recurre a esta expresión, por debajo del $10 \%$, seguido por $A B C$ con menos de un $5 \%$ y por último se encuentra El Mundo con una presencia alrededor del $2 \%$. 
Tal y como se ha comentado, las categorías "Rigidez protocolaria”, "Romper el protocolo" y "Cumplir el protocolo" se podrían englobar en una sola ya que el trasfondo es el mismo, pero también interesaba conocer hasta qué punto la prensa utiliza expresiones tan manidas como "Cumplir con el protocolo", "Romper el protocolo", "Saltarse el protocolo", "Como manda el protocolo”, porque podría guardar una estrecha relación con el hecho de que también sean las que más a menudo se utilizan en nuestra sociedad para hablar de nuestro objeto de estudio.

Categorías de nuestra clasificación en cuanto a relación terminológica se refiere como "se atribuye al protocolo algo que no le corresponde", "rigidez protocolaria" o "romper el protocolo" en donde El Mundo tiene la mayor presencia de los tres periódicos analizados, confirman que la explicación que lleva a cabo de sus códigos, no es el correcto. Así como que ostente la última posición en la categoría "correcta representación del protocolo" demuestra que la a representación que este periódico lleva a cabo del protocolo, es ciertamente negativa.

\subsection{Connotación}

Una vez explicadas todas las categorías que describen cómo es la representación del protocolo en la prensa, se finalizará con una última que cierra y resume todas lo expuesto hasta ahora, la connotación que se sustrae del protocolo en cada noticia por la forma en que viene representado.

Tabla V. Tabla de frecuencia: Connotación

\begin{tabular}{|l|l|c|c|c|c|}
\hline & & Frecuencia & Porcentaje & Porcentaje válido & Porcentaje acumulado \\
\hline \multirow{4}{*}{ Válidos } & Negativa & 197 & 63,8 & 63,8 & 63,8 \\
\cline { 2 - 6 } & Neutral & 67 & 21,7 & 21,7 & 85,4 \\
\cline { 2 - 6 } & Positiva & 45 & 14,6 & 14,6 & 100,0 \\
\cline { 2 - 6 } & Total & 309 & 100,0 & 100,0 & \\
\hline
\end{tabular}

Fuente: Elaboración propia

Se observa que la connotación que adjudica al protocolo unos valores que se perciben como negativos representa el 63,8\% de la muestra analizada, frente al $\mathbf{1 4 , 6 \%}$ de la muestra que lo hace de forma positiva para el protocolo, es decir que reconoce sus cualidades como la eficaz herramienta que es. La neutralidad también está presente en la muestra, ya que un $21,7 \%$ no adjudica ningún tipo de valor al protocolo.

Datos que se corresponden totalmente con el anterior índice sobre relaciones terminológicas, en el que el total de las categorías que podríamos denominar "negativas" asciende al 70,9\% del total ("Se atribuye al protocolo algo que no le 
corresponde”, "Rigidez protocolaria”, "Romper el protocolo”, “Gastos innecesarios”, "Puro formalismo”, “cumplir el protocolo”), y la única que consideramos positiva es "correcta representación del protocolo", que asciende al 29,9\% del total. Evidentemente no coinciden los porcentajes al 100\% porque en esta clasificación se incluye la connotación neutral, en la que se ubican todas aquellas noticias de las que no se sustrae ninguna connotación del protocolo, ni positiva ni negativa.

\section{Conclusiones}

A continuación se detallarán las conclusiones específicas que se han obtenido del trabajo de campo, para posteriormente terminar con las generales extraídas del total de la investigación.

\subsection{Conclusiones específicas}

Se comprueba que los resultados obtenidos no se corresponden con la realidad más actual del protocolo, ya que reflejan, de manera eminentemente mayoritaria, el protocolo oficial (y a un alto nivel), cuando en realidad existen muchos otros ámbitos en donde existe protocolo y cada vez, de una manera más activa, tanto en el plano empresarial, deportivo, universitario, religioso, como en el plano social.

Por un lado, se asocia el protocolo con gastos innecesarios (comidas, cócteles, regalos, viajes, etc.) algo que, por otra parte, no es estrictamente protocolo, y por otro, la misma sociedad considera el protocolo como algo propio de familias reales, poder, jerarquía, etiqueta, saber estar, glamour, azafatas, corbatas, trajes largos y un sinfín de conceptos cuanto menos, negativos para la disciplina.

Lo que sí está claro es que el concepto "gastos de protocolo" no es correcto: está mal empleado, e induce a asociar como concepto de "protocolo" algo que no se corresponde en absoluto con la realidad. Esta mala definición contribuye a desprestigiar una profesión cuya efectividad y productividad está demostrada, y que incluso en estos tiempos de crisis se está imponiendo como estrategia de marketing en las grandes empresas, por encima de la publicidad.

Asimismo, se ha corroborado cómo la prensa española sigue reflejando lo que podríamos denominar "el protocolo de ayer", relacionándolo constantemente con el poder, gran rigidez y un pomposo ceremonial. Igualmente ha quedado más que probado que el desconocimiento y el "maltrato" que esta disciplina sufre en la sociedad es directamente proporcional al que experimenta en los medios, más concretamente en la prensa.

\subsection{Conclusiones generales}

Es evidente que estamos en momentos difíciles para el protocolo por la asociación que de su término se hace a gastos, poder, glamour, mundo oficial, hecho que hemos verificado con nuestra investigación. También es evidente que el des- 
conocimiento que la sociedad tiene de la disciplina, genera un claro desinterés por el tema con obvias repercusiones negativas como el menosprecio de una profesión que todavía no ha logrado encontrar su sitio en la sociedad española.

Tema que no deja de llamar la atención, que una disciplina con tanto recorrido histórico y que cumple un papel vital en muchos ámbitos de nuestra sociedad, siga siendo reflejada en la prensa española como "algo casi obsoleto".

Existe, por tanto, un gran desconocimiento de lo que es la profesión de protocolo y eventos, del importante trabajo que realizan sus profesionales, que son los primeros en tratar de conseguir que la organización de un evento sea la adecuada o que la atención protocolizada de una institución no sean un gasto, sino una inversión por la cantidad de valor añadido aporta.

El protocolo debe considerarse una inversión rentable y duradera de las administraciones, organizaciones y empresas en tres aspectos:

- Como elementos intrínsecamente ligados a la correcta difusión de la imagen de las entidades.

- $\quad$ Como generadores de empleo al precisarse, en muchos casos, servicios externos.

- Como servicio necesario para la correcta consecución de los objetivos previstos con cada tipo de acto.

Hemos visto cómo Fuente (2008:50) definía el "protocolo de ayer" como "aquel que pretendía reflejar el poder de las autoridades mediante un pomposo ceremonial y una rígida etiqueta", frente al "protocolo de hoy" como aquel que "pretende alcanzar, armoniosamente, una buena organización y transmitir comunicación cuando el acto es pensado para llegar más allá del mero foro de invitados presentes". Este protocolo premia la organización como aspecto esencial, que flexibiliza las precedencias y las normativas vigentes, así como los usos y costumbres tradicionales, y que valora singularmente la comunicación, por lo tanto, el concepto que hemos visto reflejado en la prensa, dista mucho de ser el correcto.

Se coincide, asimismo, con Cabezuelo (2013) ${ }^{11}$ ya que no se puede juzgar al protocolo como tal, sino como protocolo + eventos, lo que se traduce en la puesta en escena del protocolo actual.

En definitiva, el protocolo que se defiende en este trabajo es una herramienta de gestión y de la excelencia en la comunicación pública y privada, que atiende a la necesidad de alcanzar la eficiencia y la perfección de las acciones humanas, y en donde la puesta en escena se convierte en fundamental. Esencia conceptual muy alejada de la que refleja, día tras día, la prensa española.

11 "Los cambios y avances que vivimos en plena globalización y la necesidad que tienen tanto las instituciones (empresas y organizaciones públicas o privadas...) y promotores de actos de lograr la más alta rentabilidad a la hora de invertir en la organización, producción y organización de eventos que promocionen y proyecten su imagen e identidad, han forzado el nacimiento del llamado Protocolo integral o Protocolo transversal." 
Ciertamente, el estudio científico nos ha demostrado que la representación que la prensa hace del protocolo, dista mucho de ser el correcto, desvirtuando en la mayoría de ocasiones su verdadera esencia conceptual. Es más, se ha comprobado que la explicación que hacen de los códigos del protocolo es incorrecto e incluso perjudicial, en la mayoría de los casos. Igualmente, ha quedado evidenciado, a través de numerosas teorías, que los medios pueden llegar a ser verdaderos creadores de opinión pública.

Por lo tanto, y llegados a este punto, estamos en disposición de refutar la hipótesis principal que se plantea al comienzo de este trabajo: La representación que la prensa española hace de los códigos del protocolo no se corresponde con su verdadera razón de ser. Una afirmación que conlleva comprobadas consecuencias negativas para la imagen de esta disciplina en la sociedad, infravalorada e incluso menospreciada, en muchos de los casos.

\section{Referencias bibliográficas}

Batlle, P; San Juan, J.C. y Sunyé, J. (2007): Buenas maneras. Madrid: Editorial Planeta.

Berrocal, S. y Rodríguez-Maribona, C. (1998): Análisis básico de la prensa diaria. Madrid: Editorial Universitas. SL.

Borau, E. (2013): “La imagen del protocolo a través de la prensa española”, Estudios sobre el Mensaje Periodístico, vol.19, pp. 633-369. Madrid: Servicio de Publicaciones de la Universidad Complutense de Madrid.

Browne, R; Del Valle, C.; Silva, V.; Carvajal, J. y Inzulza, Á. (2011): “Propuesta teórico-metodológica para un análisis crítico y complejo del discurso (ACCD) en la prensa de Chile y Perú”, Estudios sobre el Mensaje Periodístico, vol. 17, pp.17-42. Madrid: Servicio de Publicaciones de la Editorial Complutense

Cabezuelo, F. (2013): De Eventos en la Sociedad Digital. Madrid: Editorial Cersa.

Fuente, C. (2008): Protocolo Oficial. Madrid: Ediciones Protocolo.

Fuente, C. (2012): "Cuestión de protocolo". ABC, p. 16, 25-03-12.

Laforet, J. J. (1997): Protocolo y medios de comunicación social. Gran Canarias: Tegrarte SL.

Link, J. (1983): Kollektivsymbolik und Mediendiskurse. KuluRRevolution, en Wodak R. y Meyer, M. (2003): Métodos de análisis crítico del discurso. Barcelona: Editorial Gedisa.

Marín Calahorro, F. (1997): Fundamentos del protocolo en la comunicación institucional. Madrid: Editorial Síntesis.

Otero Alvarado, M.T (2004): El perfil del profesional del Protocolo. Exigencias, formación, acceso a la profesión y unificación de criterios en categorías. V Congreso Internacional de Protocolo. Febrero. Madrid.

Sierra, J. (2008): “Protocolo: Herramienta comunicativa persuasiva y simbólica”, ZER, vol. 13, n. 24, pp.337-361, País Vasco: Universidad del País Vasco. 
Tuchman, G. (1978): "Making News. A study in the construction of reality”, en VAN DIJK, Teun (1990): La noticia como discurso. Comprensión, estructura y producción de la información. Barcelona: Editorial Paidós.

Vilarrubias, F. (2010): Tratado de Protocolo: Nacional e internacional. Oviedo: Ediciones Nobel.

Wodak, R. y Meyer, M. (2003): Métodos de análisis crítico del discurso. Barcelona: Editorial Gedisa. 